\title{
Analysis of a delayed SIR model subject to multiple infectious stages and nonlinear incidence rate
}

\author{
Hong Zhang ${ }^{\mathrm{a}}$, Chunming $\mathrm{Li}^{\mathrm{b}}$, Hongquan Sun ${ }^{\mathrm{b}, *}$ \\ a School of Mathematical Science, Harbin Normal University, Harbin 150025, China. \\ ${ }^{b}$ School of Mathematical Science, Heilongjiang University, Harbin 150080, China.
}

Communicated by A. Atangana

\begin{abstract}
We investigate the threshold dynamics problem of a delayed Susceptible-Infected-Recovered (SIR) model with general nonlinear incidence and multiple parallel infectious stages. Biologically, the model contains the following aspects:

(i) once infection occurs, a fraction of the infected individuals is detected and treated, while the rest of the infected remains undetected and untreated;

(ii) distributed delays governed by a general nonlinear incidence function are included into the model due to the complexity of disease transmissions.

Mathematically, under some suitable assumptions on nonlinear incidence rate, we prove that the reproduction number $\mathfrak{R}_{0}$ can be used to govern the the global dynamics of the model. The proofs of global attractivity of disease-free equilibrium (which means the extinction of disease) and endemic equilibrium (which means the persistence of the disease) are achieved by constructing suitable Lyapunov functionals. (C)2017 All rights reserved.
\end{abstract}

Keywords: SIR epidemic model, nonlinear incidence, global attractivity, Lyapunov functional.

2010 MSC: 34D23, 34K20, 92D30.

\section{Introduction}

In the study of infectious diseases dynamics, mathematical models have been contributing to understand the mechanism of disease transmission and develop preventive measures to control infection spread. A number of mathematical models have been proposed by many researchers (see, $[8-13,15,17,18]$ and the references therein). The most basic and classic model in this field is the SIR-type epidemic model, which is governed by a system of three-dimensional ordinary differential equations (ODEs). The host population is divided into three disjoint classes such as suceceptibles, infectives and recovereds. Denote by $S(t), I(t)$ and $R(t)$ the population sizes of susceptible, infective, and recovered classes, at time $t$, respectively.

Based on SIR-type epidemic model, many studies have shown that suitable extensions could be a more appropriate way of modeling specific disease from biological significance and mathematical perspective. These extensions have been enriching our knowledge on the disease spread and control in many

\footnotetext{
*Corresponding author

Email addresses: 28812965@qq.com (Hong Zhang), lichunming@hlju.edu.cn (Chunming Li), sunhongquan@hlju.edu.cn (Hongquan Sun)

doi:10.22436/jnsa.010.11.42
} 
circumstances. Further, it is realized that more and more mathematical theory and methods have been improved to be effective tools in performing the analysis to the proposed models. Biologically, time delay and nonlinear incidence rate are two major extensions in epidemiological aspects.

- When formulated the spread of an infectious disease transmitted by a vector (for example, mosquitoes) after a specified duration (the time during which the infectious agents develop in the vector), a discrete delay $\tau$ was usually incorporated to account for the progression of a disease from latent to infective to occur, as is not the case for ODEs [2]. Moreover, according to some distribution of the vectors' latency periods, one can formulate a model with a distributed delay (see, e.g., [18]). Both of these extensions lead to a system of delay differential equations (DDEs) formulation.

- During the disease transmission dynamics, nonlinearity in the incidence rates has been observed and studied by many authors. Usually, the standard bilinear incidence rate is suggested to be modified into a nonlinear incidence rate (see, e.g., $[9,10,15])$. Changing the form of the incidence function can potentially affect and change the dynamical behaviour of the system of ODEs and DDEs. There are several different nonlinear transmission functions, we refer to the following examples and references therein: saturating incidence $\beta S \frac{\mathrm{I}}{1+\mathrm{cI}}$ (see, e.g., [1, 18]); standard incidence $\beta \frac{\mathrm{SI}}{\mathrm{N}}$ (see, e.g. [7]); separable incidence form $F(S) G(I)$ for discrete delay (see, e.g. [8]). In [8], the authors studied an SIR model, where the delay is included in $\mathrm{G}(\mathrm{I})$, modelling vector transmission, and also an SEIR system where the delay appears in both $F(S)$ and $G(I)$, modelling a fixed duration of latency. This incidence rate, $F(S) G(I)$, has been studied by Korobeinikov and Maini [11].

In a recent work, McCluskey [15] further extended and studied the Volterra-type Lyapunov functions to DDEs, resolving the global stability of the following SIR DDEs with more general incidence functions.

$$
\left\{\begin{array}{l}
\frac{d S(t)}{d t}=\lambda-\mu_{S} S(t)-\beta \int_{0}^{h} k(\tau) F(S(t), I(t-\tau)) d \tau, \\
\frac{d I(t)}{d t}=\beta \int_{0}^{h} k(\tau) F(S(t), I(t-\tau)) d \tau-\left(\mu_{I}+\gamma\right) I(t), \\
\frac{d R(t)}{d t}=\gamma I(t)-\mu_{R} R(t),
\end{array}\right.
$$

where $\lambda$ is the recruitment rate of the population. $\mu_{S}, \mu_{I}$ and $\mu_{R}$ are the death rates of susceptible individuals, infectious individuals and recovered individuals, respectively. $1 / \gamma$ represents the average time spent in class I before recovery (or removal). Thus, the total exit rate for the infected is $\mu_{\mathrm{I}}+\gamma$. It is assumed that $\mu_{\mathrm{I}}+\gamma \geqslant \mu_{\mathrm{S}}$ from biological reasons. Following method of [6], the vectors can be decoupled by including a distributed delay $\tau$ in the incidence rate for a maximum delay $h>0$ due to the fact that transmission of the disease through vectors may undergo fast dynamics.

$$
\beta \int_{0}^{h} k(\tau) F(S(t), I(t-\tau)) d \tau,
$$

represents the incidence rate at time $t$ (where $k$ is a Lebesgue integrable function). It is assumed that $\int_{0}^{h} k(\tau) d \tau=1$ for suitable $\beta$. Under some suitable assumptions on nonlinear incidence function $F, M_{c}-$ Cluskey [15] obtained the standard threshold dynamics in the sense that: the disease-free equilibrium is globally asymptotically stable for basic reproduction number being less than one and the endemic equilibrium is globally asymptotically stable for basic reproduction number exceeding one.

However, for many infectious diseases, and in particular for the diseases with a prolonged infectious period, some infectious individuals can be properly detected and treated, isolated or removed, whereas others remain undetected and untreated. Korobeinikov [10] studied this issue to consider whether length of the infectious periods and the levels of contagiousness for these detected and undetected hosts can significantly affect the system dynamics. In a subsequent work, Wang and Liu [17] extended the results of Korobeinikov [10] into the following model by adding a distributed delay to SIR model with $n$ alternative infectious pathways and $n$ noninteracting infective subclasses $I_{i}, i=1,2, \cdots, n$. The model studied in [17] takes the following form: 


$$
\left\{\begin{array}{l}
\frac{d S(t)}{d t}=\lambda-\mu S(t)-\sum_{j=1}^{n} \beta_{j} \int_{0}^{h} k_{j}\left(\tau_{j}\right) S(t) I_{j}\left(t-\tau_{j}\right) d \tau_{j}, \\
\frac{d I_{i}(t)}{d t}=p_{i} \sum_{j=1}^{n} \beta_{j} \int_{0}^{h} k_{j}\left(\tau_{j}\right) S(t) I_{j}\left(t-\tau_{j}\right) d \tau_{j}-\delta_{i} I_{i}(t), \\
\frac{d R_{i}(t)}{d t}=\sum_{j=1}^{n} r_{j} I_{j}(t)-\sigma_{i} R_{i}(t), \quad i=1,2, \cdots, n .
\end{array}\right.
$$

The parameters of model (1.2) are biologically explained as in Table 1.

Table 1: Parameters and their biological meaning in model (1.2). All these constants are assumed to be positive.

\begin{tabular}{cl}
\hline Parameter & \multicolumn{1}{c}{ Interpretation } \\
\hline \hline$p_{i} \in(0,1)$ & $\begin{array}{l}\text { the probability for an infected individual entering } \\
\text { to the } i \text {-th infective compartment; }\end{array}$ \\
$\mu$ & natural death rate of the susceptible individuals; \\
$r_{i}$ & recovery rate of infected individuals; \\
$\delta_{i}$ & the rate of the infectious individuals of the $i$-th compartment \\
& leave this compartment; \\
$\sigma_{i}$ & the remove rate of the recovered individuals.
\end{tabular}

Motivated by these works, we consider a delayed SIR epidemic model with general nonlinear incidence and multiple parallel infectious stages. In this paper, we extend model in (1.1) from a framework to one with multiple parallel infectious stages and in (1.2) to one with general nonlinear incidence. Both extensions allow for an event that intrinsic growth rate of susceptible individuals takes a general function. The main focus of this article is to investigate how multiple parallel infectious stages and general nonlinear incidence affect disease's dynamics through studying the global dynamics of our model. We define the basic reproductive number $\mathfrak{R}_{0}$ and show that it can completely determine the global dynamics of model. Under some suitable assumptions on nonlinear incidence rate and general growth rate, the existence and uniqueness of an endemic equilibrium are ensured. The approach used here to consider the effect of a wide class of nonlinear incidence rates and distributed delays is based on exploring Lyapunov functional techniques, which was used by Goh for ODEs models in ecology [4] and was known to be an effective tool to resolve the global analysis of many other epidemic models including $[12,13,15]$.

The rest of this article is organized as follows: In Section 2, a delayed SIR model of infectious disease with general nonlinear incidence and multiple parallel infectious stages is described. The preliminary results, including the definition of basic reproduction number, the existence of endemic equilibrium and well-posedness of model are presented in Section 3. Section 4 is devoted to the stability analysis of equilibria through constructing Lyapunov functionals, where we prove that the sharp threshold properties of the model rely only on the basic reproduction number $\mathfrak{R}_{0}$. In Section 5, we provide a discussion of our results and future problem related to this topic to consider.

\section{The model and preliminaries}

Based on the models given in (1.1) and (1.2), we suppose that the dynamics of the disease transmission is governed by the following system of DDEs:

$$
\left\{\begin{array}{l}
\frac{d S(t)}{d t}=N(S(t))-\sum_{j=1}^{n} \beta_{j} \int_{0}^{h} k_{j}\left(\tau_{j}\right) F\left(S(t), I_{j}\left(t-\tau_{j}\right)\right) d \tau_{j}, \\
\frac{d I_{i}(t)}{d t}=p_{i} \sum_{j=1}^{n} \beta_{j} \int_{0}^{h} k_{j}\left(\tau_{j}\right) F\left(S(t), I_{j}\left(t-\tau_{j}\right)\right) d \tau_{j}-\delta_{i} I_{i}(t), \\
\frac{d R_{i}(t)}{d t}=\sum_{j=1}^{n} r_{j} I_{j}(t)-\sigma_{i} R_{i}(t), \quad i=1,2, \cdots, n .
\end{array}\right.
$$


In view of the methods in [10] and [17], the vector classes can be omitted from the equations by incorporated a distributed delay $\tau_{j}$ in the incidence term up to a maximum delay $h>0$. The incidence at time $t$ is $\beta_{j} \int_{0}^{h} k_{j}\left(\tau_{j}\right) F\left(S(t), I_{j}\left(t-\tau_{j}\right)\right) d \tau_{j}$ (here $k_{j}(\cdot)$ is a Lebesgue integrable function). One can choose $\beta_{j}$ such that $\int_{0}^{h} k_{\mathfrak{j}}\left(\tau_{\mathfrak{j}}\right) \mathrm{d} \tau_{\mathfrak{j}}=1$.

The variables and the other parameters are defined the same as in (1.2). Since $R_{\mathfrak{i}}(t)$ does not appear in the equations for $S(t)$ and $I_{i}(t)$, it is sufficient to consider only the first two equations (2.1):

$$
\left\{\begin{array}{l}
\frac{d S(t)}{d t}=N(S(t))-\sum_{j=1}^{n} \beta_{j} \int_{0}^{h} k_{j}\left(\tau_{j}\right) F\left(S(t), I_{j}\left(t-\tau_{j}\right)\right) d \tau_{j}, \\
\frac{d I_{i}(t)}{d t}=p_{i} \sum_{j=1}^{n} \beta_{j} \int_{0}^{h} k_{j}\left(\tau_{j}\right) F\left(S(t), I_{j}\left(t-\tau_{j}\right)\right) d \tau_{j}-\delta_{i} I_{i}(t), \quad i=1,2, \cdots, n .
\end{array}\right.
$$

The initial condition for system (2.2) is

$$
S(0) \in \mathbb{R}_{+}, \quad I_{i}(\theta)=\phi_{\mathfrak{i}}(\theta), \text { for } \theta \in[-h, 0],
$$

where $\phi_{i} \in C=C\left([-h, 0], \mathbb{R}_{+}\right)$, the space of continuous functions from $[-h, 0]$ to $\mathbb{R}_{+}$, equipped with the sup-norm, $\|\phi\|=\sup _{\theta \in[-h, 0]} \phi(\theta)$.

The standard theory of functional differential equations [6] implies that the solutions of system (2.2) with initial condition (2.3) exist and are differentiable for all $t>0$. Moreover, the phase space $X=$ $\mathbb{R}_{+} \times \mathrm{C} \times \cdots \times \mathrm{C}$ is positively invariant.

On the basis of biological considerations, we make the following basic assumptions for the intrinsic growth rate of susceptible individuals $N(S(t))$.

$\left(\mathrm{A}_{1}\right) \mathrm{N}(\cdot)$ are $\mathrm{C}^{1}$ non-increasing function on $[0, \infty)$ with $\mathrm{N}(0)>0$, and there is a unique positive solution $\xi=S_{0}$ for the equation $N(\xi)=0 . N(S(t))>0$ for $0 \leqslant S(t)<S_{0}$, and $N(S(t))<0$ for $S(t)>S_{0}$, that is

$$
\left[N(S(t))-N\left(S_{0}\right)\right]\left(S(t)-S_{0}\right)<0, \text { for } S(t) \neq S_{0} .
$$

It is well-known that the class of $N(S(t))$ satisfying $\left(A_{1}\right)$ includes the situation, $N(S(t))=\lambda-d S(t)$, which has been widely used in the literature of population dynamics.

Lemma 2.1. For initial conditions in (2.3) with $S(0) \in \mathbb{R}_{+}$and $\phi_{i}(\theta) \in C$, the solutions of system (2.2) are nonnegative and ultimately uniformly bounded in $\mathrm{X}$.

Proof. First, we prove that $S(t) \geqslant 0$ for all $t \geqslant 0$. Assume for the contrary, let $t_{1}>0$ be such that $S\left(t_{1}\right)<0$. Set $t_{2}=\inf \left\{0<t<t_{1}: S(t)<0\right\}$. Then $S\left(t_{2}\right)=0$. From the first equation of system (2.2), we have $\left.\frac{\mathrm{d} S(t)}{\mathrm{dt}}\right|_{t=t_{2}}=N(0)>0$. Hence $S(t)<0$ for $t \in\left(t_{2}-\epsilon, t_{2}\right)$, for sufficiently small $\epsilon>0$. This contradicts $S(t) \geqslant 0$ for $t \in\left(0, t_{2}\right]$. It follows that $S(t) \geqslant 0$ for $t \geqslant 0$.

Next, we will show that $I_{i}(t)>0$ for all $t \geqslant 0, i=1,2, \cdots, n$. It is easy to see that disease is initially presented when the initial condition $\mathrm{I}_{i}\left(\theta_{0}\right)>0$ for some $\theta_{0} \in[-h ; 0]$, by the continuity of initial condition, $I_{i}(t)$ is positive on some interval about $\theta_{0}$. It follows that either $I_{i}(t)$ is positive for some $t \in\left[0, \theta_{0}+h\right]$ or $\frac{d I_{i}\left(\theta_{0}+h\right)}{d t}>0$, then there exists $t_{3}>0$ such that $I_{i}\left(t_{3}\right)>0$ for $t \in\left[t_{3}, t_{3}+h\right]$. From the second equation of (2.2), we have $\frac{d I_{i}(t)}{d t} \geqslant-\delta_{i} I_{i}(t)$, for $t \geqslant t_{3}$. Thus we have

$$
I_{i}(t) \geqslant I_{i}\left(t_{3}\right) e^{-\delta_{i}\left(t-t_{3}\right)}>0, \text { for } t \geqslant t_{3} .
$$

Assumption $\left(A_{1}\right)$ and the first equation of (2.2) imply that $\limsup _{t \rightarrow \infty} S(t) \leqslant S_{0}$. For each $i$, adding the two equations of (2.2) gives

$$
\frac{d S(t)}{d t}+\frac{d I_{i}(t)}{d t}=N(S)-\left(1-p_{i}\right) \sum_{j=1}^{n} \beta_{j} \int_{0}^{h} k_{j}\left(\tau_{j}\right) F\left(S(t), I_{j}\left(t-\tau_{j}\right)\right) d \tau_{j}-\delta_{i} I_{i}(t)
$$




$$
\begin{aligned}
& \leqslant m-\delta_{i} I_{i}(t) \\
& \leqslant 2 m-d\left(S(t)+I_{i}(t)\right),
\end{aligned}
$$

where

$$
p_{i} \in(0,1), \quad m=\sup _{S \in\left[0, S_{0}\right]} N(S) \text { and } d=\min \left\{\delta_{i}, \frac{m}{S_{0}}\right\}
$$

Hence,

$$
\limsup _{t \rightarrow \infty}\left(S(t)+I_{i}(t)\right) \leqslant \frac{2 m}{d} .
$$

Therefore, $S(t)$ and $I_{i}(t)$ are ultimately uniformly bounded in $X$.

Lemma 2.1 implies that omega limit sets of system (2.2) are contained in the following bounded feasible region:

$$
\Gamma=\left\{\left(\mathrm{S}(\mathrm{t}), \mathrm{I}_{\mathrm{i}}(\mathrm{t})\right) \in \mathrm{X}: 0<\mathrm{S}(\mathrm{t}) \leqslant \mathrm{S}_{0}, 0<\mathrm{S}(\mathrm{t})+\mathrm{I}_{\mathrm{i}}(\mathrm{t}) \leqslant \frac{2 \mathrm{~m}}{\mathrm{~d}}, \mathrm{i}=1,2, \cdots, \mathrm{n}\right\} .
$$

It can be verified that the region $\Gamma$ is positively invariant with respect to model (2.2) and the model is well-posed. Our results in this paper will be stated for system (2.2) in $\Gamma$.

\section{Equilibria and $\mathfrak{R}_{0}$}

Under the assumption $\left(A_{1}\right)$, system (2.2) has the disease-free equilibrium $E_{0}=\left(S_{0}, 0, \cdots, 0\right)$ on the boundary of $\Gamma$. The partial derivatives of $F$ are denoted by $F_{1}$ and $F_{2}$ in what follows.

The basic reproduction number for the model (2.2) is

$$
\mathfrak{R}_{0}=\sum_{i=1}^{n} \frac{\beta_{i} p_{i}}{\delta_{i}} F_{2}\left(E_{0}\right),
$$

which is defined as the expected number of new infections caused by a single newly infected individual in an entirely susceptible population during its entire infectious period [3].

An equilibrium $E^{*}=\left(S^{*}, I_{1}^{*}, I_{2}^{*}, \cdots, I_{n}^{*}\right)$ in the interior $\Gamma$ is called an endemic equilibrium. Note that the equilibria of system (2.2) are the same as the equilibria of the corresponding ODEs system. Thus $S^{*}, I_{i}^{*}>0$ satisfies the following algebraic equations:

$$
\left\{\begin{array}{l}
0=N\left(S^{*}\right)-\sum_{j=1}^{n} \beta_{j} F\left(S^{*}, I_{j}^{*}\right), \\
0=\sum_{j=1}^{n} \beta_{j} F\left(S^{*}, I_{j}^{*}\right)-\frac{\delta_{i}}{p_{i}} I_{i}^{*} .
\end{array}\right.
$$

Adding the two equations, we have $N\left(S^{*}\right)-\frac{\delta_{i}}{p_{i}} I_{i}^{*}=0$, and so $I_{i}^{*}=\frac{p_{i} N\left(S^{*}\right)}{\delta_{i}}$. Let

$$
H\left(S^{*}\right)=\sum_{j=1}^{n} \beta_{j} F\left(S^{*}, \frac{p_{j} N\left(S^{*}\right)}{\delta_{j}}\right)-N\left(S^{*}\right) .
$$

Then $\frac{d I_{i}(t)}{d t}=0$ whenever $H\left(S^{*}\right)=0$. Thus, any zero of $H(\cdot)$ in the interval $\left(0, S_{0}\right)$ corresponds to an equilibrium with $S^{*}, I_{i}^{*}>0$, that is, an endemic equilibrium. It follows from $F\left(0, I_{i}\right)=F(S, 0)=0$ that $\mathrm{H}(0)=-\mathrm{N}(0)<0$ and $\mathrm{H}\left(\mathrm{S}_{0}\right)=0$. The function $\mathrm{H}(\cdot)$ is continuous. It follows that a sufficient condition for $\mathrm{H}(\cdot)$ obtaining a zero solution in $\left(0, S_{0}\right)$ is that $\mathrm{H}(\cdot)$ is increasing at $S_{0}$. Thus, an endemic equilibrium exists if the following condition is fulfilled

$$
0<\left.\frac{\mathrm{dH}}{\mathrm{dS}}\right|_{\left(S=S_{0}\right)}=\sum_{j=1}^{n} \beta_{j} F_{1}\left(E_{0}\right)+\left.\sum_{j=1}^{n} \beta_{j} F_{2}\left(E_{0}\right) \frac{p_{j}}{\delta_{j}} \frac{d N(S)}{d S}\right|_{\left(S=S_{0}\right)}-\left.\frac{d N(S)}{d S}\right|_{\left(S=S_{0}\right)} .
$$

Since $F(S, 0)=0$ for all $S$, it follows that $F_{1}\left(E_{0}\right)=0$ and so (3.2) is equivalent to $\mathfrak{R}_{0}>1$. Hence we are in a 
position to state the following result.

Lemma 3.1. If $\mathfrak{R}_{0}>1$, then system (2.2) admits an endemic equilibrium $\mathrm{E}^{*}=\left(\mathrm{S}^{*}, \mathrm{I}_{1}^{*}, \mathrm{I}_{2}^{*}, \cdots, \mathrm{I}_{\mathrm{n}}^{*}\right)$.

\section{Stability analysis of (2.1) by Lyapunov functional techniques}

It is commonly realized that the rigorous proofs of the global dynamics depending on the basic reproduction number $\mathfrak{R}_{0}$ are nontrivial for many disease models [16]. In particular, due to the complexity and high dimension of disease models, the global stability of the endemic equilibrium is known as a challenging mathematical problem.

For models where the sharp threshold property holds, and knowledge of disease data are available, sensitivity analysis of $\mathfrak{R}_{0}$ can be used to evaluate the effects of control strategies.

In what follows, we state our main results concerning the global properties of the disease-free equilibrium $E_{0}$ and the endemic equilibrium $E^{*}$ of (2.2). $\mathfrak{R}_{0}$ is the key threshold parameter characterizing the global dynamics of (2.2), through constructing suitable the Lyapunov functionals.

4.1. Global attractivity of disease-free equilibrium for $\mathfrak{R}_{0} \leqslant 1$

Theorem 4.1. Assume that the intrinsic growth rate of susceptible individuals $\mathrm{N}(\mathrm{S}(\mathrm{t}))$, and the nonlinear incidence rate $\mathrm{F}\left(\mathrm{S}(\mathrm{t}), \mathrm{I}_{\mathrm{i}}(\mathrm{t})\right)$ satisfy the following conditions:

$\left(\mathrm{A}_{2}\right)\left(\mathrm{N}\left(\mathrm{S}_{0}\right)-\mathrm{N}(\mathrm{S}(\mathrm{t}))\right)\left[\mathrm{F}_{2}(\mathrm{~S}(\mathrm{t}), 0)-\mathrm{F}_{2}\left(\mathrm{~S}_{0}, 0\right)\right] \geqslant 0$ for all $\mathrm{S}(\mathrm{t}) \in\left(0, \mathrm{~S}_{0}\right]$ with equality only if $\mathrm{S}(\mathrm{t})=\mathrm{S}_{0}$.

$\left(\mathrm{A}_{3}\right) \mathrm{F}\left(\mathrm{S}(\mathrm{t}), \mathrm{I}_{\mathfrak{i}}(\mathrm{t})\right) \leqslant \mathrm{I}_{\mathfrak{i}}(\mathrm{t}) \mathrm{F}_{2}(\mathrm{~S}(\mathrm{t}), 0)$ for all $\mathrm{S}(\mathrm{t}), \mathrm{I}_{\mathfrak{i}}(\mathrm{t}) \in \Gamma$.

If $\mathfrak{R}_{0} \leqslant 1$, the disease-free equilibrium of system $(2.2), \mathrm{E}_{0}=\left(\mathrm{S}_{0}, 0, \cdots, 0\right)$ is globally attracting in $\mathrm{X}$.

Proof. Let

$$
G(x)=x-S_{0}-\int_{S_{0}}^{x} \frac{F_{2}\left(S_{0}, 0\right)}{F_{2}(\sigma, 0)} d \sigma
$$

then $\frac{d G(x)}{d x}=1-\frac{F_{2}\left(S_{0}, 0\right)}{F_{2}(x, 0)}$. It follows from $\left(A_{2}\right)$ that its sign changes from non-positive to non-negative as $x$ increases through $S_{0}$. Thus, $G(x)$ has global minimum value at $S_{0}$ with $G\left(S_{0}\right)=0$, that is, $G(x) \geqslant 0$ for all $x \geqslant 0$.

Define $K_{j}\left(\tau_{j}\right)=\int_{\tau_{j}}^{h} k_{j}(s) d s$. Then it follows from the support of $k_{j}$ having positive measure near $h$ that $K_{j}\left(\tau_{j}\right)>0$ for $0 \leqslant \tau_{j}<h$. Hence it is easy to see that $K_{j}(h)=0$. We construct the Lyapunov functional for $\mathrm{E}_{0}$ as follows,

$$
\mathcal{L}_{\text {DFE }}(t)=G(S(t))+\sum_{i=1}^{n} a_{i} I_{i}(t)+F_{2}\left(S_{0}, 0\right) U_{+}(t),
$$

where

$$
\left\{\begin{array}{l}
u_{+}(t)=\sum_{j=1}^{n} \beta_{j} \int_{0}^{h} K_{j}\left(\tau_{j}\right) I_{j}\left(t-\tau_{j}\right) d \tau_{j}, \\
a_{i}=\frac{\beta_{i}}{\delta_{i}} F_{2}\left(S_{0}, 0\right), \quad i=1,2, \cdots, n .
\end{array}\right.
$$

It follows from $(4.1)$ and $I_{i}(t) \geqslant 0$ that $U_{+}(t) \geqslant 0$ with equality if and only if $I_{i}(t)$ is identically zero on the interval $[t-h, t]$.

Direct calculating and using integration by parts yield

$$
\frac{d U_{+}(t)}{d t}=-\sum_{j=1}^{n} \beta_{j} \int_{0}^{h} K_{j}\left(\tau_{j}\right) \frac{d}{d \tau_{j}} I_{j}\left(t-\tau_{j}\right) d \tau_{j}
$$




$$
\begin{aligned}
& =-\left.\sum_{j=1}^{n} \beta_{j} K_{j}\left(\tau_{j}\right) I_{j}\left(t-\tau_{j}\right)\right|_{\tau_{j}=0} ^{h}+\sum_{j=1}^{n} \int_{0}^{h} \beta_{j} I_{j}\left(t-\tau_{j}\right) d K_{j}\left(\tau_{j}\right) \\
& =\sum_{j=1}^{n} \beta_{j} \int_{0}^{h} k_{j}\left(\tau_{j}\right) I_{j}(t) d \tau_{j}-\sum_{j=1}^{n} \int_{0}^{h} \beta_{j} I_{j}\left(t-\tau_{j}\right) k_{j}\left(\tau_{j}\right) d \tau_{j} \\
& =\sum_{j=1}^{n} \beta_{j} \int_{0}^{h} k_{j}\left(\tau_{j}\right)\left[I_{j}(t)-I\left(t-\tau_{j}\right)\right] d \tau_{j},
\end{aligned}
$$

which will be used in estimating Lyapunov functional $\mathcal{L}_{\mathrm{DFE}}(\mathrm{t})$. By (4.2), we obtain the time derivative of $\mathcal{L}_{\mathrm{DFE}}(\mathrm{t})$ along the solutions of system (2.2) as follows

$$
\begin{aligned}
\left.\frac{\mathrm{d} \mathcal{L}_{D F E}(t)}{d t}\right|_{(2.2)}= & {\left[1-\frac{F_{2}\left(S_{0}, 0\right)}{F_{2}(S(t), 0)}\right] N(S(t))-\sum_{j=1}^{n} \beta_{j} \int_{0}^{h} k_{j}\left(\tau_{j}\right) F\left(S(t), I_{j}\left(t-\tau_{j}\right)\right) d \tau_{j} } \\
& +\frac{F_{2}\left(S_{0}, 0\right)}{F_{2}(S(t), 0)} \sum_{j=1}^{n} \beta_{j} \int_{0}^{h} k_{j}\left(\tau_{j}\right) F\left(S(t), I_{j}\left(t-\tau_{j}\right)\right) d \tau_{j} \\
& +\sum_{i=1}^{n} a_{i} p_{i} \sum_{j=1}^{n} \beta_{j} \int_{0}^{h} k_{j}\left(\tau_{j}\right) F\left(S(t), I_{j}\left(t-\tau_{j}\right)\right) d \tau_{j}-\sum_{i=1}^{n} a_{i} \delta_{i} I_{i}(t) \\
& +F_{2}\left(S_{0}, 0\right) \sum_{j=1}^{n} \beta_{j} \int_{0}^{h} k_{j}\left(\tau_{j}\right)\left[I_{j}(t)-I\left(t-\tau_{j}\right)\right] d \tau_{j} \\
= & \left.N(S(t))-N\left(S_{0}\right)\right]\left[1-\frac{F_{2}\left(S_{0}, 0\right)}{F_{2}(S(t), 0)}\right]-\sum_{i=1}^{n} a_{i} \delta_{i} I_{i}(t) \\
& +\left[\sum_{i=1}^{n} a_{i} p_{i}-1\right] \sum_{j=1}^{n} \beta_{j} \int_{0}^{h} k_{j}\left(\tau_{j}\right) F\left(S(t), I_{j}\left(t-\tau_{j}\right)\right) d \tau_{j} \\
& +F_{2}\left(S_{0}, 0\right) \sum_{j=1}^{n} \beta_{j} \int_{0}^{h} k_{j}\left(\tau_{j}\right)\left[\frac{F\left(S(t), I_{j}\left(t-\tau_{j}\right)\right)}{F_{2}(S(t), 0)}+I_{j}(t)-I_{j}\left(t-\tau_{j}\right)\right] d \tau_{j} .
\end{aligned}
$$

Recall that

$$
\mathfrak{R}_{0}=\sum_{i=1}^{n} \frac{\beta_{i} p_{i}}{\delta_{i}} F_{2}\left(S_{0}, 0\right)=\sum_{i=1}^{n} a_{i} p_{i}
$$

Rearranging $\left.\frac{\mathrm{d} \mathcal{L}_{\mathrm{DFE}}(\mathrm{t})}{\mathrm{dt}}\right|_{(2.2)}$ yields

$$
\begin{aligned}
\left.\frac{\mathrm{d} \mathcal{L}_{\text {DFE }}(t)}{d t}\right|_{(2.2)}= & -\left[N\left(S_{0}\right)-N(S(t))\right]\left[1-\frac{F_{2}\left(S_{0}, 0\right)}{F_{2}(S(t), 0)}\right]-\sum_{i=1}^{n} \beta_{i} I_{i}(t) F_{2}\left(S_{0}, 0\right) \\
& +\left(\Re_{0}-1\right) \sum_{j=1}^{n} \beta_{j} \int_{0}^{h} k_{j}\left(\tau_{j}\right) F\left(S(t), I_{j}\left(t-\tau_{j}\right)\right) d \tau_{j}+\sum_{i=1}^{n} \beta_{i} I_{i}(t) F_{2}\left(S_{0}, 0\right) \\
& +F_{2}\left(S_{0}, 0\right) \sum_{j=1}^{n} \beta_{j} \int_{0}^{h} k_{j}\left(\tau_{j}\right)\left[\frac{F\left(S(t), I_{j}\left(t-\tau_{j}\right)\right)}{F_{2}(S(t), 0)}-I_{j}\left(t-\tau_{j}\right)\right] d \tau_{j} \\
= & -\left[N\left(S_{0}\right)-N(S(t))\right]\left[1-\frac{F_{2}\left(S_{0}, 0\right)}{F_{2}(S(t), 0)}\right]
\end{aligned}
$$




$$
\begin{aligned}
& +\left(\Re_{0}-1\right) \sum_{j=1}^{n} \beta_{j} \int_{0}^{h} k_{j}\left(\tau_{j}\right) F\left(S(t), I_{j}\left(t-\tau_{j}\right)\right) d \tau_{j} \\
& +F_{2}\left(S_{0}, 0\right) \sum_{j=1}^{n} \beta_{j} \int_{0}^{h} k_{j}\left(\tau_{j}\right)\left[\frac{F\left(S(t), I_{j}\left(t-\tau_{j}\right)\right)}{F_{2}(S(t), 0)}-I_{j}\left(t-\tau_{j}\right)\right] d \tau_{j} .
\end{aligned}
$$

Then, it follows from assumptions $\left(\mathrm{A}_{2}\right)$ and $\left(\mathrm{A}_{3}\right)$ that

$$
\left\{\begin{array}{l}
-\left[N\left(S_{0}\right)-N(S(t))\right]\left[1-\frac{F_{2}\left(S_{0}, 0\right)}{F_{2}(S(t), 0)}\right] \leqslant 0 \\
F_{2}\left(S_{0}, 0\right) \sum_{j=1}^{n} \beta_{j} \int_{0}^{h} k_{j}\left(\tau_{j}\right)\left[\frac{F\left(S(t), I_{j}\left(t-\tau_{j}\right)\right)}{F_{2}(S(t), 0)}-I_{j}\left(t-\tau_{j}\right)\right] d \tau_{j} \leqslant 0
\end{array}\right.
$$

Denote $\mathcal{A}_{0}=\left\{\left.\frac{\mathrm{d} \mathcal{L}_{\mathrm{DFE}}(\mathrm{t})}{\mathrm{dt}}\right|_{(2.2)}=0\right\}$ and let $\mathcal{M}_{0}$ be the largest invariant set in $\mathcal{A}_{0}$. If $\left.\frac{\mathrm{d} \mathcal{L}_{\mathrm{DFE}}(\mathrm{t})}{\mathrm{dt}}\right|_{(2.2)} \leqslant 0$, one can apply Lyapunov-LaSalle Theorem [6, Theorem 5.3.1] to system (2.2) and immediately arrives at the following result: every omega limit set is contained in $\mathcal{M}_{0}$.

In fact, when $\mathfrak{R}_{0}<1$, from assumptions $\left(\mathrm{A}_{2}\right)$ and $\left(\mathrm{A}_{3}\right)$, we immediately have the following result,

$$
\left.\frac{\mathrm{d} \mathcal{L}_{\mathrm{DFE}}(\mathrm{t})}{\mathrm{dt}}\right|_{(2.2)} \leqslant\left(\mathfrak{R}_{0}-1\right) \sum_{j=1}^{n} \beta_{j} \int_{0}^{h} k_{j}\left(\tau_{j}\right) F\left(S(t), I_{j}\left(t-\tau_{j}\right)\right) d \tau_{j} \leqslant 0,
$$

with equality only if $S(t)=S^{0}$ or $I_{i}(t)=0$, for $i=1,2, \cdots, n$. So $\mathcal{M}_{0}$ contains only the singleton $\left\{E_{0}\right\}$. Thus $\mathrm{E}_{0}$ is globally attracting.

When $\mathfrak{R}_{0}=1$, from assumptions $\left(\mathrm{A}_{4}\right)$ and $\left(\mathrm{A}_{5}\right)$, we immediately have the following result,

$$
\begin{aligned}
\left.\frac{\mathrm{d} \mathcal{L}_{\text {DFE }}(\mathrm{t})}{\mathrm{dt}}\right|_{(2.2)}= & -\left[\mathrm{N}\left(\mathrm{S}_{0}\right)-\mathrm{N}(\mathrm{S}(\mathrm{t}))\right]\left[1-\frac{\mathrm{F}_{2}\left(\mathrm{~S}_{0}, 0\right)}{\mathrm{F}_{2}(\mathrm{~S}(\mathrm{t}), 0)}\right] \\
& +\mathrm{F}_{2}\left(\mathrm{~S}_{0}, 0\right) \sum_{j=1}^{n} \beta_{j} \int_{0}^{h} k_{j}\left(\tau_{j}\right)\left[\frac{F\left(S(t), I_{j}\left(t-\tau_{j}\right)\right)}{F_{2}(S(t), 0)}-I_{j}\left(t-\tau_{j}\right)\right] d \tau_{j} .
\end{aligned}
$$

Hence,

$$
\left\{\begin{array}{l}
\left.\frac{\mathrm{d} \mathcal{L}_{\mathrm{DFE}}(\mathrm{t})}{\mathrm{dt}}\right|_{(2.2)} \leqslant-\left[\mathrm{N}\left(\mathrm{S}_{0}\right)-\mathrm{N}(\mathrm{S}(\mathrm{t}))\right]\left[1-\frac{\mathrm{F}_{2}\left(\mathrm{~S}_{0}, 0\right)}{\mathrm{F}_{2}(\mathrm{~S}(\mathrm{t}), 0)}\right], \text { or } \\
\left.\frac{\mathrm{d} \mathcal{L}_{\mathrm{DFE}}(\mathrm{t})}{\mathrm{dt}}\right|_{(2.2)} \leqslant \mathrm{F}_{2}\left(\mathrm{~S}_{0}, 0\right) \sum_{j=1}^{n} \beta_{j} \int_{0}^{h} k_{j}\left(\tau_{j}\right)\left[\frac{F\left(S(t), I_{j}\left(t-\tau_{j}\right)\right)}{F_{2}(S(t), 0)}-I_{j}\left(t-\tau_{j}\right)\right] \mathrm{d} \tau_{j} .
\end{array}\right.
$$

Both of the cases, we can verify that $\mathcal{M}_{0}$ consists of only the singleton $\left\{\mathrm{E}_{0}\right\}$. Thus $\mathrm{E}_{0}$ is globally attracting. Then if $\mathfrak{R}_{0} \leqslant 1$, the disease-free equilibrium of system $(2.2), E_{0}=\left(S_{0}, 0, \cdots, 0\right)$, is globally attracting in $X$. This completes the proof.

\subsection{Global attractivity of endemic equilibrium for $\mathfrak{R}_{0}>1$}

In this subsection, as a precondition, we assume that $\mathfrak{R}_{0}>1$, guaranteeing the existence of an endemic equilibrium $E^{*}=\left(S^{*}, I_{1}^{*}, I_{2}^{*}, \cdots, I_{\mathrm{n}}^{*}\right)$ (see Lemma 3.1). We obtain the global dynamics of endemic equilibrium for $\Re_{0}>1$, provided that certain assumptions on $\mathrm{N}(\mathrm{S}(\mathrm{t}))$, and $\mathrm{F}$ are satisfied.

Theorem 4.2. Assume that $\mathfrak{R}_{0}>1$ and endemic equilibrium $\mathrm{E}^{*}$ exists. Suppose functions $\mathrm{N}(\mathrm{S}(\mathrm{t})), \mathrm{F}\left(\mathrm{S}(\mathrm{t}), \mathrm{I}_{\mathfrak{i}}(\mathrm{t})\right)$ satisfy the following conditions:

$\left(A_{4}\right) \operatorname{sgn}\left(F\left(S(t), I_{\mathfrak{i}}^{*}\right)-F\left(S^{*}, I_{\mathfrak{i}}^{*}\right)\right)=\operatorname{sgn}\left(N(S(t))-N\left(S^{*}\right)\right)$ for all $S(t)>0$.

$\left(A_{5}\right) \frac{F\left(S(t), I_{i}(t)\right)}{F\left(S(t), I_{i}^{*}\right)}$ is in the closed interval with endpoints at 1 and $\frac{I(t)}{I^{*}}$ for all $S(t), I_{i}(t)>0$. 
Then the endemic equilibrium $\mathrm{E}^{*}$ is globally attracting in $\mathrm{X}$.

Proof. From Lemma 3.1, an endemic equilibrium $E^{*}$ exists if $\mathfrak{R}_{0}>1$. In what follows we prove that $E^{*}$ is globally attracting. Consider the Volterra function

$$
\mathrm{g}(z)=z-1-\ln z \geqslant 0, \quad z \in \mathbb{R}_{+} .
$$

Thus $g(z) \geqslant 0$ for all $z>0$, and has global minimum with $g(z)=0$ if and only if $z=1$.

Let $b_{i}=\frac{\beta_{i} F\left(S^{*}, I_{i}^{*}\right)}{\delta_{i} I_{i}^{*}}$ and define a Lyapunov functional for $E^{*}$ of system (2.2),

$$
\mathcal{L}_{\mathrm{EE}}(\mathrm{t})=\mathrm{L}_{\mathrm{S}}(\mathrm{t})+\mathrm{L}_{\mathrm{I}}(\mathrm{t})+\mathrm{V}_{+}(\mathrm{t}),
$$

where

$$
\left\{\begin{array}{l}
L_{S}(t)=S(t)-S^{*}-\int_{S^{*}}^{S(t)} \frac{F\left(S^{*}, I_{j}^{*}\right)}{F\left(\sigma, I_{j}^{*}\right)} d \sigma \\
L_{I}(t)=\sum_{i=1}^{n} b_{i} I_{i}^{*} g\left(\frac{I_{i}(t)}{I_{i}^{*}}\right), \\
V_{+}(t)=\sum_{i=1}^{n} \int_{0}^{h} \beta_{i} K_{i}\left(\tau_{i}\right) F\left(S^{*}, I_{i}^{*}\right) g\left(\frac{I_{i}\left(t-\tau_{i}\right)}{I_{i}^{*}}\right) d \tau_{i} .
\end{array}\right.
$$

We begin by differentiating the function $\frac{d V_{+}(t)}{d t}$, which will be used later to obtain $\frac{d \mathcal{L}_{E E}(t)}{d t}$. Using integration by parts, we have

$$
\begin{aligned}
\left.\frac{d V_{+}(t)}{d t}\right|_{(2.2)}= & -\sum_{i=1}^{n} \beta_{i} \int_{0}^{h} K_{i}\left(\tau_{i}\right) F\left(S^{*}, I_{i}^{*}\right) d g\left(\frac{I_{i}\left(t-\tau_{i}\right)}{I_{i}^{*}}\right) \\
= & -\left.\sum_{i=1}^{n} \beta_{i} K_{i}\left(\tau_{i}\right) F\left(S^{*}, I_{i}^{*}\right) g\left(\frac{I_{i}\left(t-\tau_{i}\right)}{I_{i}^{*}}\right)\right|_{\tau_{i}=0} ^{h} \\
& +\sum_{i=1}^{n} \beta_{i} \int_{0}^{h} F\left(S^{*}, I_{i}^{*}\right) g\left(\frac{I_{i}\left(t-\tau_{i}\right)}{I_{i}^{*}}\right) d K_{i}\left(\tau_{i}\right) \\
= & \sum_{i=1}^{n} \beta_{i} \int_{0}^{h} k_{i}\left(\tau_{i}\right) F\left(S^{*}, I_{i}^{*}\right)\left[g\left(\frac{I_{i}(t)}{I_{i}^{*}}\right)-g\left(\frac{I_{i}\left(t-\tau_{i}\right)}{I_{i}^{*}}\right)\right] d \tau_{i} .
\end{aligned}
$$

Calculating the time derivative of $\mathcal{L}_{\mathrm{EE}}(\mathrm{t})$ along the solutions of (2.2) gives

$$
\begin{aligned}
\left.\frac{\mathrm{d} \mathcal{L}_{E E}(t)}{\mathrm{dt}}\right|_{(2.2)}= & -\left[N\left(S^{*}\right)-N(S(t))\right]\left[1-\frac{F\left(S^{*}, I_{j}^{*}\right)}{F\left(S(t), I_{j}^{*}\right)}\right] \\
& -\sum_{i=1}^{n} \beta_{i} \int_{0}^{h} k_{i}\left(\tau_{i}\right) F\left(S(t), I_{j}\left(t-\tau_{j}\right)\right) d \tau_{j}+N\left(S^{*}\right)\left[1-\frac{F\left(S^{*}, I_{j}^{*}\right)}{F\left(S(t), I_{j}^{*}\right)}\right] \\
& +\frac{F\left(S^{*}, I_{j}^{*}\right)}{F\left(S(t), I_{j}^{*}\right)} \sum_{j=1}^{n} \beta_{j} \int_{0}^{h} k_{j}\left(\tau_{j}\right) F\left(S(t), I_{j}\left(t-\tau_{j}\right)\right) d \tau_{j} \\
& +\sum_{i=1}^{n} b_{i} p_{i} \sum_{j=1}^{n} \beta_{j} \int_{0}^{h} k_{j}\left(\tau_{j}\right) F\left(S(t), I_{j}\left(t-\tau_{j}\right)\right) d \tau_{j}-\sum_{i=1}^{n} b_{i} p_{i} \delta_{i} I_{i}(t) \\
& -\sum_{i=1}^{n} b_{i} p_{i} \frac{I_{i}^{*}}{I_{i}} \sum_{j=1}^{n} \beta_{j} \int_{0}^{h} k_{j}\left(\tau_{j}\right) F\left(S(t), I_{j}\left(t-\tau_{j}\right)\right) d \tau_{j}+\sum_{i=1}^{n} b_{i} p_{i} \delta_{i} I_{i}^{*}
\end{aligned}
$$




$$
+\sum_{i=1}^{n} \beta_{i} \int_{0}^{h} k_{i}\left(\tau_{i}\right) F\left(S^{*}, I_{i}^{*}\right)\left[g\left(\frac{I_{i}(t)}{I_{i}^{*}}\right)-g\left(\frac{I_{i}\left(t-\tau_{i}\right)}{I_{i}^{*}}\right)\right] d \tau_{i} .
$$

From (3.1), $\sum_{i=1}^{n} b_{i} p_{i}=1$ and $b_{i} p_{i} \beta_{j} F\left(S^{*}, I_{i}^{*}\right)=b_{j} p_{j} \beta_{i} F\left(S^{*}, I_{i}^{*}\right)$, we have

$$
\begin{aligned}
\frac{\left.\mathrm{d} \mathcal{L}_{E E}(t)\right|_{(2.2)}=}{\mathrm{dt}} & -\left[\mathrm{N}\left(S^{*}\right)-N(S(t))\right]\left[1-\frac{F\left(S^{*}, I_{j}^{*}\right)}{F\left(S(t), I_{j}^{*}\right)}\right]+\sum_{j=1}^{n} \beta_{j} F\left(S^{*}, I_{i}^{*}\right) \\
& -\frac{F\left(S^{*}, I_{j}^{*}\right)}{f\left(S(t), I_{j}^{*}\right)} \sum_{j=1}^{n} \beta_{j} F\left(S^{*}, I_{i}^{*}\right) \\
& -\sum_{j=1}^{n} \beta_{j} \int_{0}^{h} k_{j}\left(\tau_{j}\right) F\left(S(t), I\left(t-\tau_{j}\right)\right) d \tau_{j} \\
& +\frac{F\left(S^{*}, I_{j}^{*}\right)}{F\left(S(t), I_{j}^{*}\right)} \sum_{j=1}^{n} \beta_{j} \int_{0}^{h} k_{j}\left(\tau_{j}\right) F\left(S(t), I\left(t-\tau_{j}\right)\right) d \tau_{j} \\
& +\sum_{j=1}^{n} b_{i} p_{i} \sum_{j=1}^{n} \beta_{j} \int_{0}^{h} k_{j}\left(\tau_{j}\right) F\left(S(t), I\left(t-\tau_{j}\right)\right) d \tau_{j}-\sum_{i=1}^{n} \beta_{i} \frac{I_{i}}{I_{i}^{*}} F\left(S^{*}, I_{i}^{*}\right) \\
& -\sum_{j=1}^{n} b_{i} p_{i} \frac{I_{i}}{I_{i}^{*}} \sum_{j=1}^{n} \beta_{j} \int_{0}^{h} k_{j}\left(\tau_{j}\right) F\left(S(t), I\left(t-\tau_{j}\right)\right) d \tau_{j}+\sum_{i=1}^{n} \beta_{i} F\left(S^{*}, I_{i}^{*}\right) \\
& +\sum_{i=1}^{n} \beta_{i} \int_{0}^{h} k_{i}\left(\tau_{i}\right) F\left(S^{*}, I_{i}^{*}\right)\left[g\left(\frac{I_{i}(t)}{I_{i}^{*}}\right)-g\left(\frac{I_{i}\left(t-\tau_{i}\right)}{I_{i}^{*}}\right)\right] d \tau_{i} \\
= & -\left[N\left(S^{*}\right)-N(S(t))\right]\left[1-\frac{F\left(S^{*}, I_{j}^{*}\right)}{F\left(S(t), I_{j}^{*}\right)}\right] \\
& +\sum_{j=1}^{n} b_{i} p_{i} \sum_{j=1}^{n} \beta_{j} \int_{0}^{h} k_{j}\left(\tau_{j}\right) F\left(S^{*}, I_{j}^{*}\right) \mathcal{M}\left(\tau_{j}\right) d \tau_{j},
\end{aligned}
$$

where

$$
\begin{aligned}
\mathcal{M}\left(\tau_{j}\right)= & 2-\frac{F\left(S^{*}, I_{j}^{*}\right)}{F\left(S(t), I_{j}^{*}\right)}+\frac{F\left(S(t), I_{j}\left(t-\tau_{j}\right)\right)}{F\left(S(t), I_{j}^{*}\right)}-\frac{I_{i}^{*} F\left(S(t), I_{j}\left(t-\tau_{j}\right)\right)}{I_{i} F\left(S^{*}, I_{j}^{*}\right)} \\
& -\frac{I_{j}\left(t-\tau_{j}\right)}{I_{j}^{*}}+\ln \frac{I_{j}\left(t-\tau_{j}\right)}{I_{j}^{*}}-\ln \frac{I_{j}(t)}{I_{j}^{*}} \\
= & -g\left(\frac{F\left(S^{*}, I_{j}^{*}\right)}{F\left(S(t), I_{j}^{*}\right)}\right)-g\left(\frac{I_{i}^{*} F\left(S(t), I_{j}\left(t-\tau_{j}\right)\right)}{I_{i} F\left(S^{*}, I_{j}^{*}\right)}\right)-g\left(\frac{I_{j}\left(t-\tau_{j}\right)}{I_{j}^{*}}\right) \\
& +g\left(\frac{F\left(S(t), I_{j}\left(t-\tau_{j}\right)\right)}{F\left(S(t), I_{j}^{*}\right)}\right)+\ln \frac{I_{i} I_{j}^{*}}{I_{i}^{*} I_{j}} .
\end{aligned}
$$

Note that

$$
\sum_{i=1}^{n} b_{i} p_{i} \sum_{j=1}^{n} \beta_{j} F\left(S^{*}, I_{j}^{*}\right) \ln \frac{I_{i} I_{j}^{*}}{I_{i}^{*} I_{j}}=\sum_{i=2}^{n} b_{i} p_{i} \sum_{j=1}^{i-1} \beta_{j} F\left(S^{*}, I_{j}^{*}\right) \ln \frac{I_{i} I_{j}^{*}}{I_{i}^{*} I_{j}}
$$




$$
\begin{aligned}
& +\sum_{i=1}^{n-1} b_{i} p_{i} \sum_{j=1+1}^{n} \beta_{j} F\left(S^{*}, I_{j}^{*}\right) \ln \frac{I_{i} I_{j}^{*}}{I_{i}^{*} I_{j}} \\
= & \sum_{i=2}^{n} b_{i} p_{i} \sum_{j=1}^{i-1} \beta_{j} F\left(S^{*}, I_{j}^{*}\right) \ln \frac{I_{i} I_{j}^{*}}{I_{i}^{*} I_{j}} \\
& +\sum_{i=2}^{n} b_{i} p_{i} \sum_{j=1}^{i-1} \beta_{j} F\left(S^{*}, I_{j}^{*}\right) \ln \frac{I_{i}^{*} I_{j}}{I_{i} I_{j}^{*}} \\
= & 0 .
\end{aligned}
$$

Similar to the arguments in [15] and due to the fact that function $g(\cdot)$ is monotone on each side of 1 and is minimized at 1 , assumption $\left(A_{5}\right)$ implies

$$
g\left(\frac{F\left(S(t), I_{j}\left(t-\tau_{j}\right)\right)}{F\left(S(t), I_{j}^{*}\right)}\right) \leqslant g\left(\frac{I_{j}\left(t-\tau_{j}\right)}{I_{j}^{*}}\right) .
$$

Since $g(\cdot) \geqslant 0$, we have $M\left(\tau_{j}\right) \leqslant 0$. Then, under the assumption $\left(\mathrm{A}_{4}\right)$, we can conclude that

$$
\left.\frac{\mathrm{d} \mathcal{L}_{\mathrm{EE}}(\mathrm{t})}{\mathrm{dt}}\right|_{(2.2)} \leqslant 0
$$

Lyapunov-LaSalle Theorem [5, Theorem 5.3.1] implies that solutions of system (2.2) tend to $\nwarrow$, the largest invariant subset of $\left\{\left.\frac{\mathrm{dL} \mathrm{LE}_{\mathrm{EE}}(\mathrm{t})}{\mathrm{dt}}\right|_{(2.2)}=0\right\}$. Thus $\left.\frac{\mathrm{d} \mathcal{L}_{\mathrm{EE}}(\mathrm{t})}{\mathrm{dt}}\right|_{(2.2)}=0$ if and only if $\mathrm{S}=\mathrm{S}^{*}$ and

$$
k_{j}\left(\tau_{j}\right)\left(\frac{I_{i}^{*} F\left(S(t), I_{j}\left(t-\tau_{j}\right)\right)}{I_{i} F\left(S^{*}, I_{j}^{*}\right)}\right),
$$

for almost every $\tau_{j} \in[0, h]$. That is $k_{j}\left(\tau_{j}\right)=0$ or $F\left(S^{*}, I_{j}\left(t-\tau_{j}\right)\right)=F\left(S^{*}, I_{j}^{*}\right) \frac{I_{i}}{I_{i}^{*}}$ holds almost everywhere. It follows from $S=S^{*}$ lies in $\aleph$ that $\frac{\mathrm{dS}(\mathrm{t})}{\mathrm{dt}}=0$ at each point in $\aleph$. That is,

$$
\begin{aligned}
0 & =N\left(S^{*}\right)-\sum_{j=1}^{n} \beta_{j} \int_{0}^{h} k_{j}\left(\tau_{j}\right) F\left(S^{*}, I_{j}\left(t-\tau_{j}\right)\right) d \tau_{j} \\
& =N\left(S^{*}\right)-\frac{I_{i}}{I_{i}^{*}} \sum_{j=1}^{n} \beta_{j} \int_{0}^{h} k_{j}\left(\tau_{j}\right) F\left(S^{*}, I_{j}^{*}\right) d \tau_{j},
\end{aligned}
$$

which requires $I_{i}$ to be constant, and in turn gives $I_{i}=I_{i}^{*}$ for all $t$. Thus, each element of $\aleph$ satisfies $S(t)=S^{*}$ and $I_{i}=I_{i}^{*}$ for all $t$. We are now in a position to conclude that $\lim _{t \rightarrow \infty}\left(S(t), I_{i}(t)\right)=\left(S^{*}, I_{i}^{*}\right)=E^{*}$. This completes the proof of globally attractivity of the endemic equilibrium $E^{*}$ if $\mathfrak{R}_{0}>1$.

Remark 4.3. Let $N(S(t))=\lambda-d S(t)$ and $F\left(S(t), I_{j}(t)\right)=S(t) I_{j}(t)$. Then system (2.2) will reduce to the model studied in [17]. The assumptions on $N(S(t))$ and $F\left(S(t), I_{j}(t)\right)=S(t) I_{j}(t),\left(A_{1}\right)-\left(A_{5}\right)$, are satisfied automatically. The disease-free equilibrium $E_{0}$ and endemic equilibrium $E^{*}$ become $E_{0}^{1}=\left(\frac{\lambda}{d}, 0, \cdots, 0\right)$ and $E^{* 1}=\left(S^{* 1}, I_{1}^{* 1}, \cdots, I_{n}^{* 1}\right)$, where $S^{* 1}=\frac{1}{\sum_{j=1}^{n} \frac{\beta_{j} p_{j}}{\delta_{j}}}$, and $I_{i}^{* 1}=\frac{\left(\lambda-d S^{* 1}\right) p_{i}}{\delta_{i}} \cdot \Re_{0}$ of system (2.2) becomes $\mathfrak{R}_{0}^{1}=\sum_{i=1}^{n} \frac{\beta_{i} p_{i}}{\delta_{i}} \frac{\lambda}{d}$.

Therefore, applying Theorems 4.1, and 4.2 to above reduced system, we immediately have the following result.

Corollary 4.4 (See [17, Theorem 3.1]). Consider system (2.2) with the case of $\mathrm{N}(\mathrm{S}(\mathrm{t}))=\lambda-\mathrm{dS}(\mathrm{t})$ and $\mathrm{F}\left(\mathrm{S}(\mathrm{t}), \mathrm{I}_{\mathrm{j}}(\mathrm{t})\right)=\mathrm{S}(\mathrm{t}) \mathrm{I}_{\mathrm{j}}(\mathrm{t})$.

(i) If $\Re_{0}^{1} \leqslant 1$, then $\mathrm{E}_{0}^{1}=\left(\frac{\lambda}{\mathrm{d}}, 0, \cdots, 0\right)$ is globally attracting.

(ii) If $\mathfrak{R}_{0}^{1}>1$, then $\mathrm{E}^{* 1}=\left(\mathrm{S}^{* 1}, \mathrm{I}_{1}^{* 1}, \ldots, \mathrm{I}_{\mathrm{n}}^{* 1}\right)$ is globally attracting whenever it exists. 


\section{Summary and discussion}

In this paper, under some suitable assumptions, the system (2.2) exhibits sharp threshold properties of the disease-free equilibrium for $\mathfrak{R}_{0} \leqslant 1$, and the endemic equilibrium for $\mathfrak{R}_{0}>1$. Our approach (Lyapunov functional techniques) has similarities to that used in Huang et al. [8], Korobeinikov [911], McCluskey [12-15], Wang and Liu [17]. Compared to [15, 17], our constructing method explicitly demonstrates how to find the Lyapunov functionals for the DDEs system of interest.

For biological motivations, we not only extend the model (1.1) to one with multiple parallel infectious stages and model (1.2) to one with general nonlinear incidence rate but also obtain the global properties for (2.2) with distributed time delays governed by a more general class of nonlinear incidence rate.

The sharp threshold properties are obtained by assuming growth rate and nonlinear incidence rate enjoy the nice properties, which is characterized by assumptions $\left(\mathrm{A}_{1}\right)-\left(\mathrm{A}_{5}\right)$. Mathematically, assumption $\left(A_{1}\right)$ ensures the existence of endemic equilibrium when $\mathfrak{R}_{0}>1$. The global attractivity of the diseasefree equilibrium requires the technical assumptions $\left(A_{2}\right)-\left(A_{3}\right)$. Results on the global properties of the endemic equilibrium for $\Re_{0}>1$ require additional assumptions on $F,\left(A_{4}\right)-\left(A_{5}\right)$. The results obtained in this paper is just global attractivity, but not global asymptotic stability. The reason for this lies in that the local asymptotic stability of the equilibria of system (2.2) have not been proved. Lyapunov-LaSalle Theorem [6, Theorem 5.3.1] only give the information that the omega limit set are contained in the largest invariance set of $\left\{\left.\frac{\mathrm{dL}_{\mathrm{DFE}}(\mathrm{t})}{\mathrm{dt}}\right|_{(2.2)}=0\right\}$ or $\left\{\left.\frac{\mathrm{dL}_{\mathrm{EE}}(\mathrm{t})}{\mathrm{dt}}\right|_{(2.2)}=0\right\}$. More detail information on the local behaviors of equilibria of system (2.2) may need extra assumptions on the form of $\mathrm{N}(\cdot)$ and $\mathrm{F}$. These will be left as our future consideration.

\section{Acknowledgment}

The authors would like to thank the editors and the referees for their helpful comments. C. Li and H. Sun are supported by Research Foundation of Heilongjiang University (No. HDJCCX-201618).

\section{References}

[1] V. Capasso, G. Serio, A generalization of the Kermack-McKendrickdeterministic epidemic model, Math. Biosci., 42 (1978), 43-61. 1

[2] K. L. Cooke, Stability analysis for a vector disease model, Rocky Mountain J. Math., 9 (1979), 31-42. 1

[3] O. Diekmann, J. A. P. Heesterbeek, J. A. J. Metz, On the definition and the computation of the basic reproduction ratio $\mathrm{R}_{0}$ in models for infectious diseases in heterogeneous populations, J. Math. Biol., 28 (1990), 365-382. 3

[4] B. S. Goh, Stability of some multispecies population models, Dekker, New York, (1980). 1

[5] J. K. Hale, J. Kato, Phase space for retarded equations with infinite delay, Funkcial. Ekvac., 21 (1978), 11-41. 4.2

[6] J. K. Hale, S. M. Verduyn Lunel, Introduction to Functional Differential Equations, Springer-Verlag, New York, (1993). $1,2,4.1,5$

[7] H. W. Hethcote, Qualitative analyses of communicable disease models, Math. Biosci., 28 (1976), 335-356. 1

[8] G. Huang, Y. Takeuchi, W. Ma, D. Wei, Global stabilty for delay SIR and SEIR epidemic models with nonlinear incidence rate, Bull. Math. Biol., 28 (2010), 1192-1207. 1, 5

[9] A. Korobeinikov, Global properties of infectious disease models with nonlinear incidence, Bull. Math. Biol., 69 (2007), 1871-1886. 1, 5

[10] A. Korobeinikov, Global properties of SIR and SEIR epidemic models with multiple parallel infectious stages, Bull. Math. Biol., 71 (2009), 75-83. 1, 1, 2

[11] A. Korobeinikov, P. K. Maini, Nonlinear incidence and stability of infectious disease models, Math. Med. Biol., 22 (2005), 113-128. 1, 5

[12] C. C. McCluskey, Global stability for an SEIR epidemiological model with varying infectivity and infinite delay, Math. Biosci. Eng., 6 (2009), 603-610. 1, 5

[13] C. C. McCluskey, Complete global stability for an SIR epidemic model with delay-distributed or discrete, Nonlinear Anal. Real World Appl., 11 (2010), 55-69. 1, 1

[14] C. C. McCluskey, Global stability for an SIR epidemic model with delay and nonlinear incidence, Nonlinear Anal. Real World Appl., 11 (2010), 3106-3109.

[15] C. C. McCluskey, Global stability of an SIR epidemic model with delay and general nonlinear incidence, Math. Biosci. Eng., 7 (2010), 837-850. 1, 1, 1, 4.2, 5 
[16] Z. Shuai, P. van en Driessche, Global stability of infectious disease models using Lyapunov functions, SIAM J. Appl. Math., 73 (2013), 1513-1532. 4

[17] X. Wang, S. Liu, Global properties of a delayed SIR epidemic model with multiple parallel infectious stages, Math. Biosci. Eng., 9 (2012), 685-695. 1, 1, 2, 4.3, 4.4, 5

[18] R. Xu, Z. Ma, Global stability of a SIR epidemic model with nonlinear incidence rate and time delay, Nonlinear Anal. Real World Appl., 10 (2009), 3175-3189. 1 